\title{
Classificação de Angle: uma sugestão de modificação pela relação sagital de caninos
}

\section{Angle classification: a suggestion for modification by the sagittal relationship of canines}

\begin{abstract}
RESUMO
A existência de variações na má oclusão entre a relação molar e a relação dos caninos, demonstra a necessidade de agrupar os problemas de maneira clara e precisa. A grande importância dos caninos na oclusão justifica o estabelecimento de uma nomenclatura própria para descrever os desvios da normalidade, contribuindo para uma comunicação entre os ortodontistas, auxiliando no prognóstico, diagnóstico, plano de tratamento e terapêutica. Portanto, o objetivo deste artigo é sugerir uma complementação a classificação de Angle com a introdução da relação sagital de caninos na análise da má oclusão. A nomenclatura, utilizada para a classificação sagital dos caninos, pode contribuir para preencher uma lacuna existente nas classificações das más oclusões quando se avalia a relação mesiodistal dos elementos dentários, proporcionando a avaliação sagital mais precisa dos pacientes.
\end{abstract}

Palavras-chave: Classificação de Angle, Má oclusão, Dentes caninos, Ortodontia

\begin{abstract}
The existence of variations in malocclusion between the molar relationship and the canine relationship, demonstrates the need to group the problems clearly and precisely. The great importance of canines in occlusion justifies the establishment of a specific nomenclature to describe deviations from normality, contributing to communication between orthodontists, helping with prognosis, diagnosis, treatment plan and therapy. Therefore, the aim of this article is to suggest a complement to Angle's classification with the introduction of the sagittal relationship of canines in the analysis of malocclusion. The nomenclature, used for the sagittal classification of canines, can contribute to fill a gap in the classification of malocclusions when assessing the mesiodistal relationship of dental elements, providing the most accurate sagittal assessment of patients.

Keywords: Angle classification, Malocclusion, Canine teeth, Orthodontics
\end{abstract}

\section{INTRODUÇÃO}

As classificações das más oclusões desempenham importante papel na Ortodontia, sendo necessárias para identificar de forma precisa, detalhada e completa os desvios da normalidade, agrupando os problemas segundo as características comuns, de maneira clara, facilitando assim, a comunicação entre os profissionais. A classificação das más oclusões contribui para o estabelecimento do diagnóstico e para a elaboração de um plano de tratamento, e o clínico é conduzido a refletir sobre a severidade do problema e a raciocinar sobre possíveis soluções ${ }^{1}$.
Augusto Alberto Foggiato ${ }^{1}$

Antônio Carlos Pereira ${ }^{2}$

Renato Castro de Almeida ${ }^{3}$

Cesar Henrique Fukuji Fuziy ${ }^{4}$

Douglas Fernandes da Silva ${ }^{5}$

Acácio Fuziy ${ }^{6}$

${ }^{1}$ Doutor em Ortodontia- São Leopoldo Mandic; Professor da Universidade Estadual do Norte do Paraná- UENP; Instituto de Pesquisa em Odontologia, Medicina e Fototerapia Foggiato. ${ }^{2}$ Doutor em Saúde Pública; Professor da Faculdade de Odontologia de Piracicaba da Universidade Estadual de Campinas - UNICAMP. ${ }^{3}$ Doutor em Ortodontia; Professor dos Cursos de Especialização em Ortodontia da ABO Osaco e SPO - São Paulo.

${ }^{4}$ Graduando em Odontologia - Faculdade de Odontologia de Araçatuba- Universidade Estadual Paulista Júlio de Mesquita Filho

${ }^{5}$ Doutor em Microbiologia Aplicada- UNESP Rio Claro; Professor da Universidade Estadual do Norte do Paraná - UENP; Instituto de Pesquisa em Odontologia, Medicina e Fototerapia Foggiato.

${ }^{6}$ Doutor e Pós-doutorado em OrtodontiaFaculdade de Odontologia de Bauru- USP, Coordenador do Curso de Especialização em Ortodontia- Orocentro- Itapetininga; Instituto de Pesquisa em Odontologia, Medicina e Fototerapia Foggiato; Pesquisador da Orthometric.

Autor para correspondência:

Nome do autor: Acácio Fuziy

Endereço: Av. São Paulo, 355- Bairro Cascata Marília- SP

Telefone: (14) 99761-1559 
De acordo com o Manual da Organização Mundial da Saúde - OMS - "Levantamentos em saúde bucal" (2017), os desvios morfológicos da oclusão normal na população mundial são tão grandes que é considerado o terceiro maior problema odontológico ${ }^{2}$. Muitas classificações das más oclusões foram apresentadas na Ortodontia, dentre estas destacam-se de Kingsley ${ }^{3}$, Angle ${ }^{4}$, Case ${ }^{5}$, Lischer ${ }^{6}$, Dewey $^{7}$, Anderson $^{8}$, Ackerman e Pro$\mathrm{ffit}^{9}, \mathrm{Katz}^{10}$. Apesar da existência de outras classificações, a terminologia criada por Angle um século atrás, continua até os dias atuais como sendo a mais universalmente aceita e que dividiu as más oclusões em três, Classe I, Classe II e Classe III, sendo que nas más oclusões de Classes II e III, quando o problema se manifesta unilateralmente, recebem a denominação de subdivisão para indicar o lado do erro ${ }^{1}$.

Em estudo realizado por Katz (1992), do qual participaram 347 especialistas em Ortodontia e que responderam questionários sobre a classificação de Angle presente em fotografias de modelos de gesso que apresentavam oclusões ideais e severidades distintas de Classe II. Observaram uma concordância baixa nas respostas dos participantes, exceto nos casos de oclusões ideais. Concluíram que o sistema de classificação de Angle é inconsistente ${ }^{1}$.

MOYERS, em 1988, afirmou que um dos erros mais comuns em Odontologia é tentar rotular cada caso imediatamente. A classificação não é um elemento único de diagnóstico, sendo sensato descrever aquilo que é observado de errado, de maneira completa e precisa, para depois enquadrar o problema dentro de uma determinada classificação. ${ }^{11}$ Variações nas posições dentárias ocorrem e se manifestam distintamente nas más oclusões, e considerando-se que nem sempre os primeiros molares possam ser os elementos principais de uma classificação, é que o presente artigo propõe uma complementação à classificação de Angle, incluindo a informação da posição sagital dos caninos, contribuindo desta forma para a elaboração do diagnóstico e planejamento dos tratamentos.

\section{MATERIAIS E MÉTODOS}

Por meio das observações clínicas, fotográficas e de modelos de gesso, Foggiato $(2002)^{12}$ propôs uma terminologia para as más oclusões fundamentada na relação sagital dos caninos.

\section{RESULTADOS}

Após a análise das imagens e com base na literatura, pode-se dividir a relação sagital dos caninos em:

Classe 1 de Canino- c1

Denomina-se de c1, quando a ponta da cúspide do canino superior ocluir na direção da ameia do canino e o primeiro pré-molar inferior. (Figura 1a).

Classe II de Canino - c2

A nomenclatura de c2 é empregada quando a ponta da cúspide do canino superior está posicionada mesialmente à ameia do canino e pré-molar inferior. (Figura 1b).

Classe 111 de Canino- c3

Classifica-se como c3, quando a ponta da cúspide do canino superior está posicionada distalmente à ameia do canino e pré-molar inferior. (Figura 1c). 


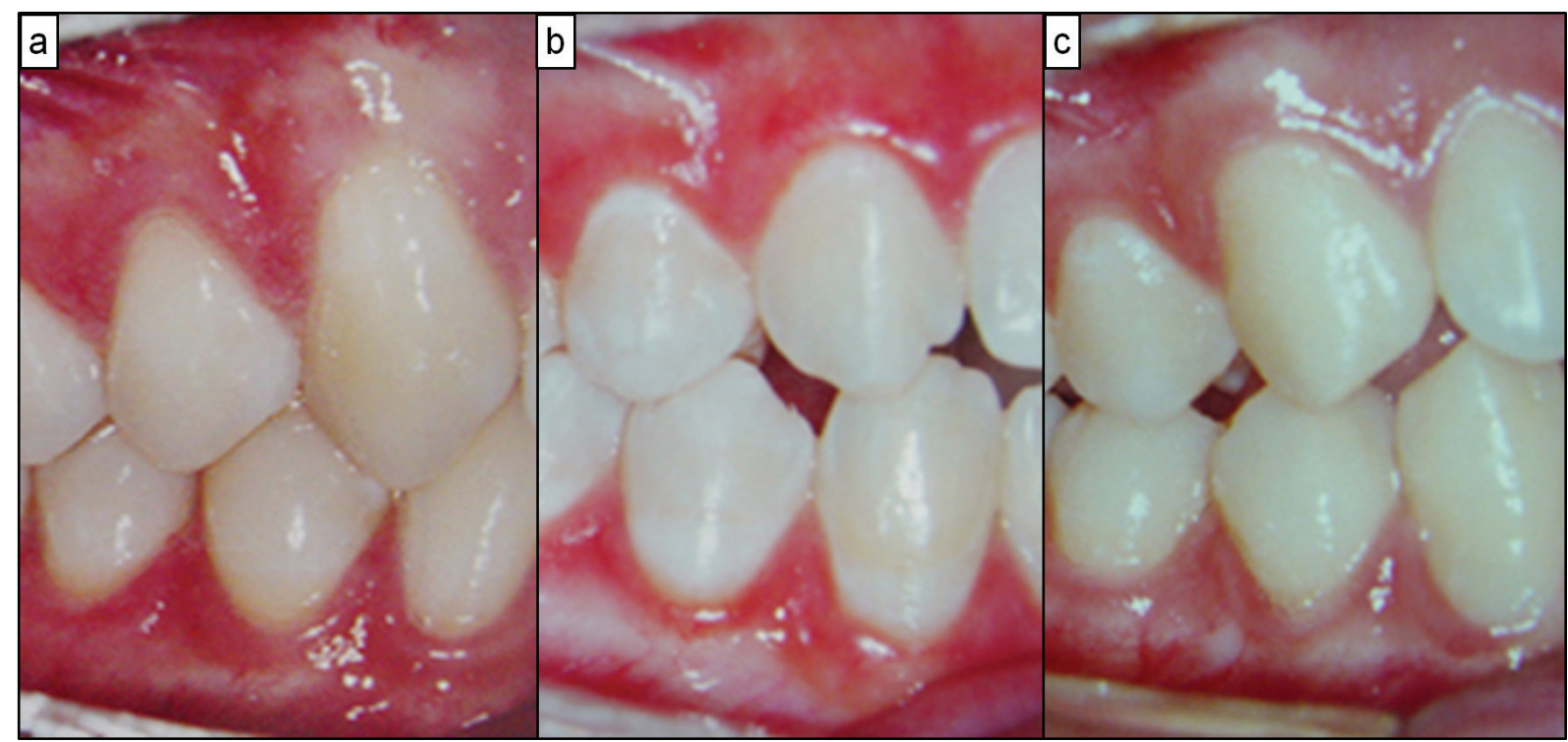

Figura 1: a) Ilustra a relação sagital de caninos c1; b) Ilustra a relação sagital de caninos c2; c) Ilustra a relação sagital de caninos c3.

\section{SUBDIVIS ÕES}

Podem ser observadas variações considerando-se os lados direito e esquerdo. Diante da situação em que um dos lados do arco dentário se enquadra em uma determinada classificação, e do lado oposto em outra, utiliza-se as letras " $R$ " para o lado direito e " $L$ " para o lado esquerdo. Exemplo de subdivisão nas Figuras 2a e 2b.

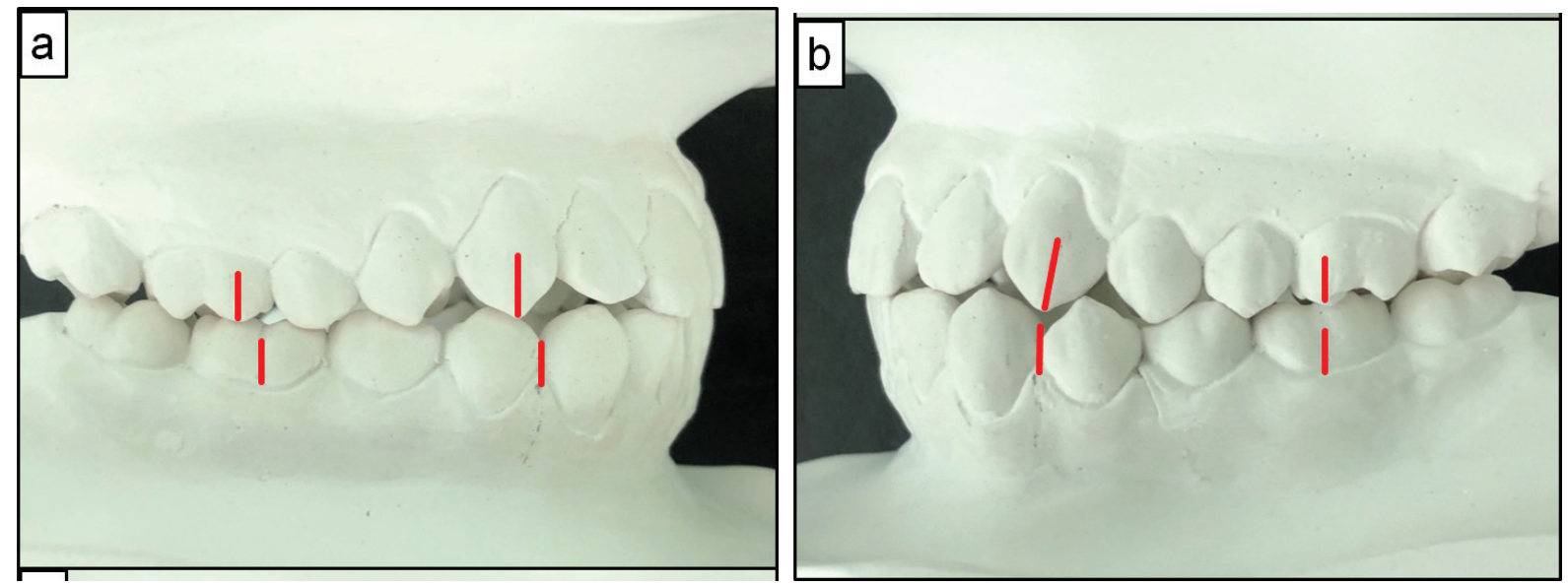

Figura 2: a) Mostra a subdivisão Classe III- c3R; b) Mostra a subdivisão Classe I- c1L.

Combinações entre a Classificação de ANGLE e a classificação sagital de caninos.

A relação de molares de Angle pode se combinar com as três possíveis relações sagitais de caninos como pode ser observado no quadro 1 e nas Figuras de 3 a 11.

\begin{tabular}{|l|ll|ll|l|}
\hline$\bullet$ Classe 1, c1; & $\bullet$ & Classe 11, div.1, c1; & $\bullet$ & Classe 11, div.2, c1; & $\bullet$ Classe 111, c1; \\
$\bullet$ Classe 1, c2; & $\bullet$ & Classe 11, div.1, c2; & $\bullet$ & Classe 11, div.2, c2; & $\bullet$ Classe 111, c2; \\
$\bullet$ Classe 1, c3; & $\bullet$ Classe 11, div.1, c3; & $\bullet \quad$ Classe 11, div.2, c3; & $\bullet$ Classe 111, c3. \\
\hline
\end{tabular}

Quadro 1: Combinação da Classificação de ANGLE com a classificação sagital de caninos. 


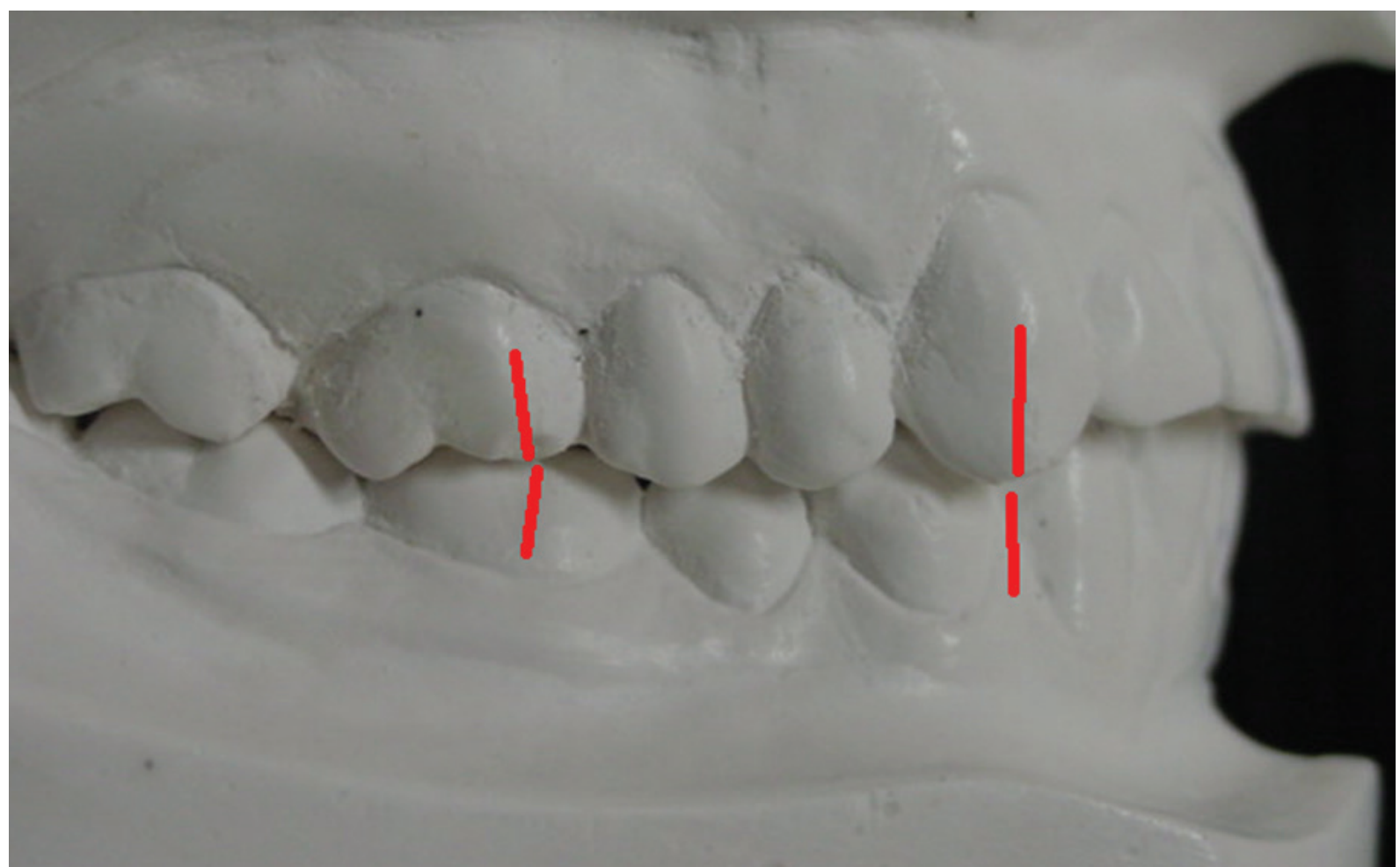

Figura 3: Evidencia a relação molar de Classe I e de caninos em c1.

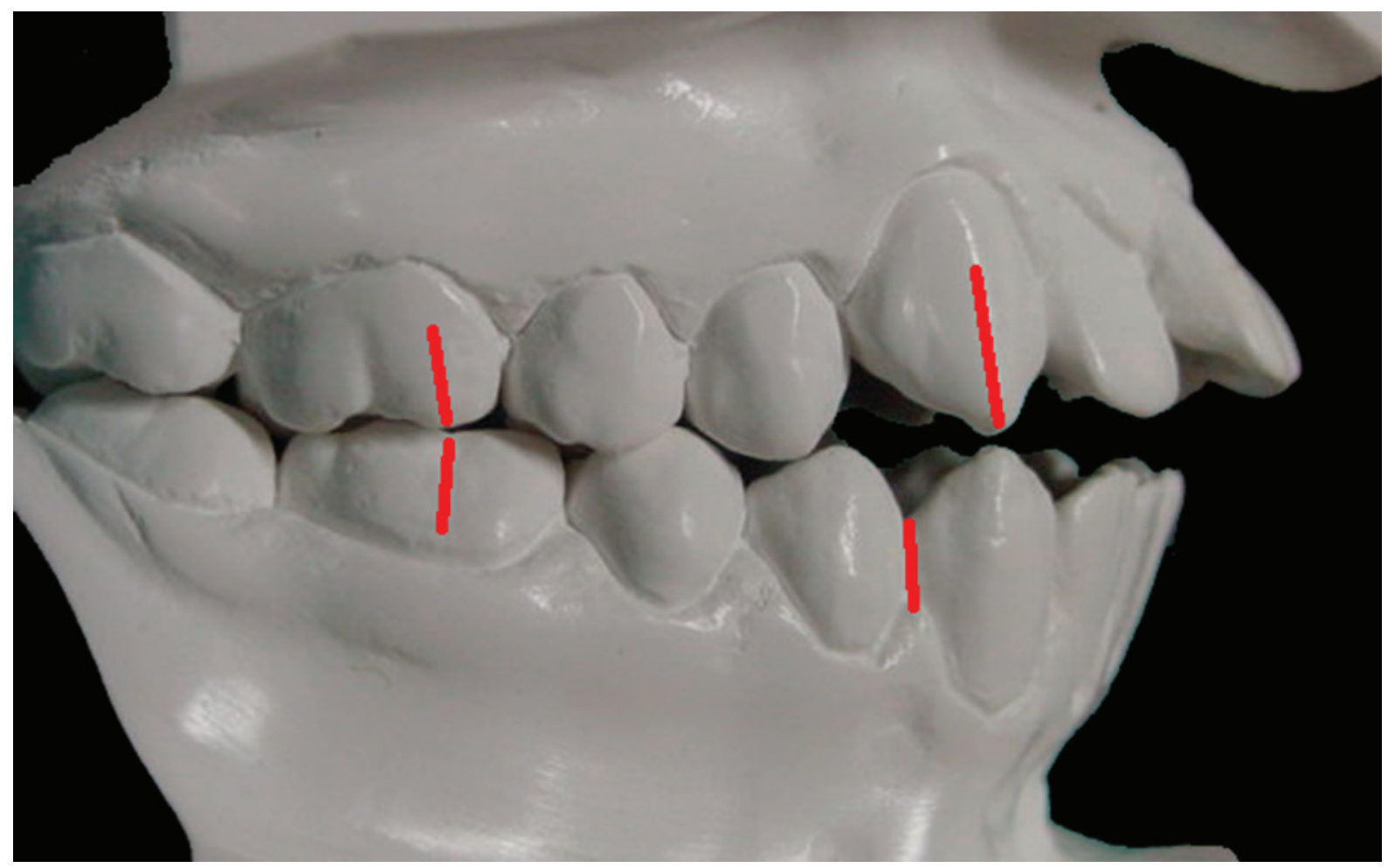

Figura 4: Evidencia a relação molar de Classe I e de caninos em c2. 


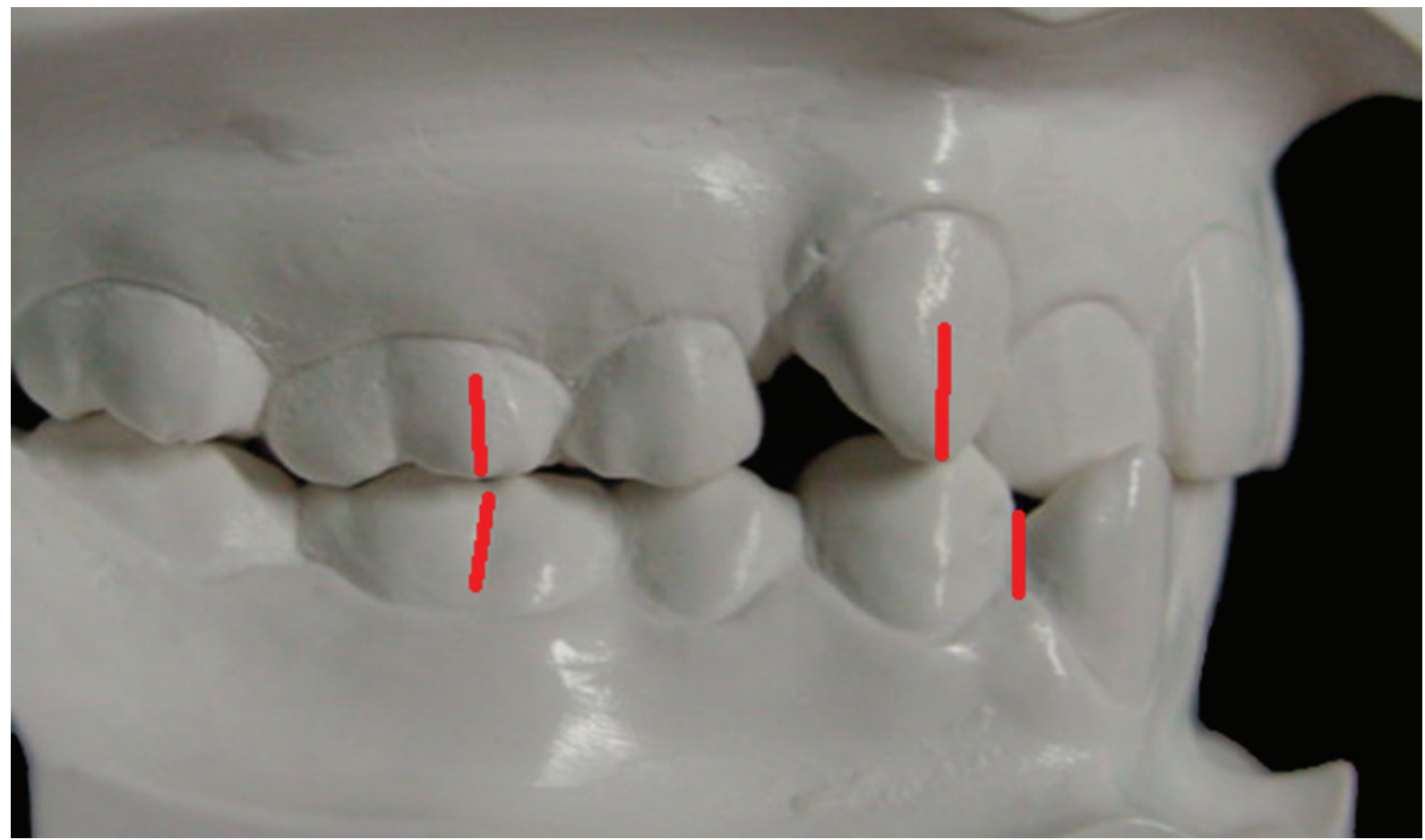

Figura 5: Evidencia a relação molar de Classe I e de caninos em c3.

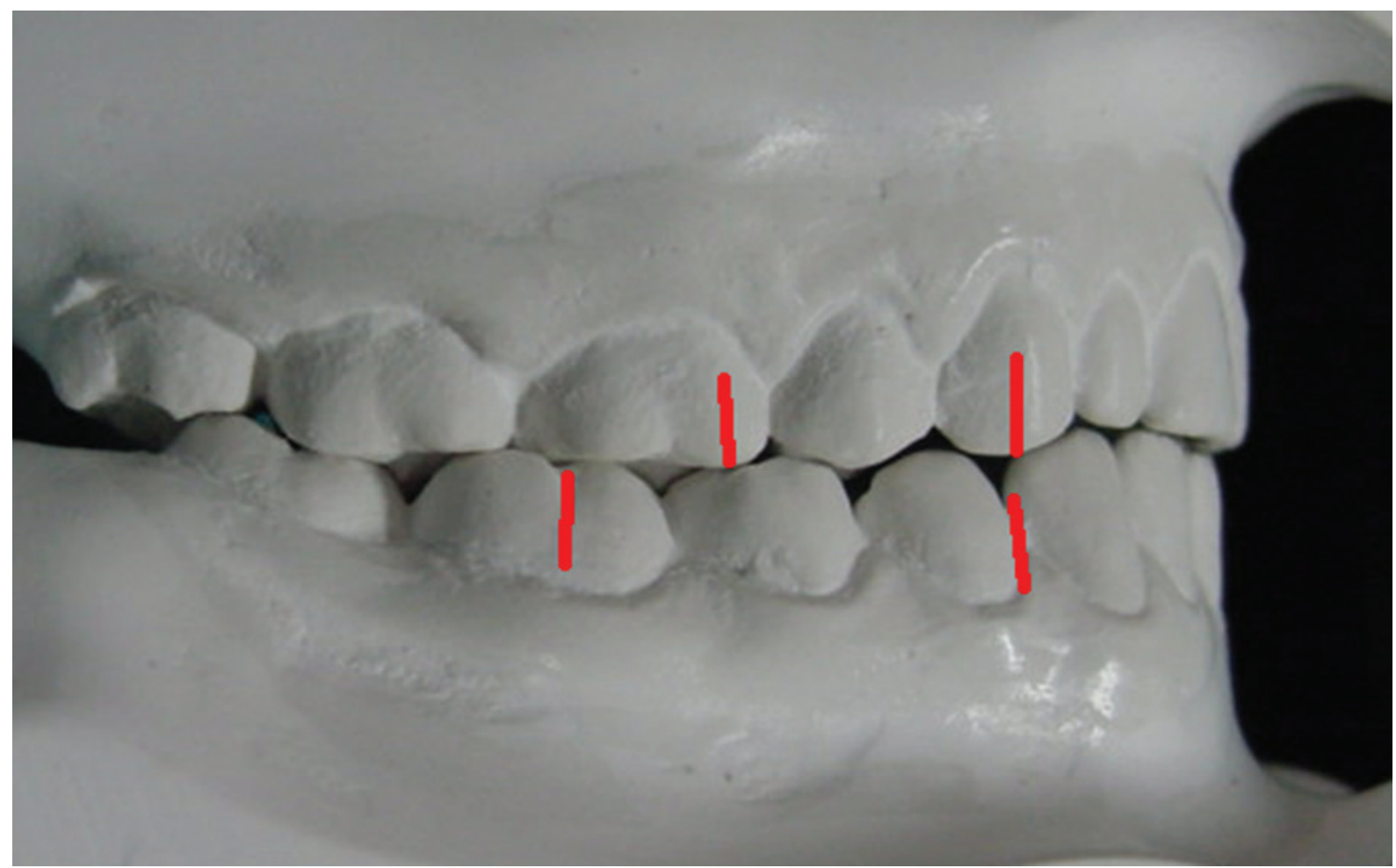

Figura 6: Apresenta a relação molar de Classe II e de caninos em c1. 


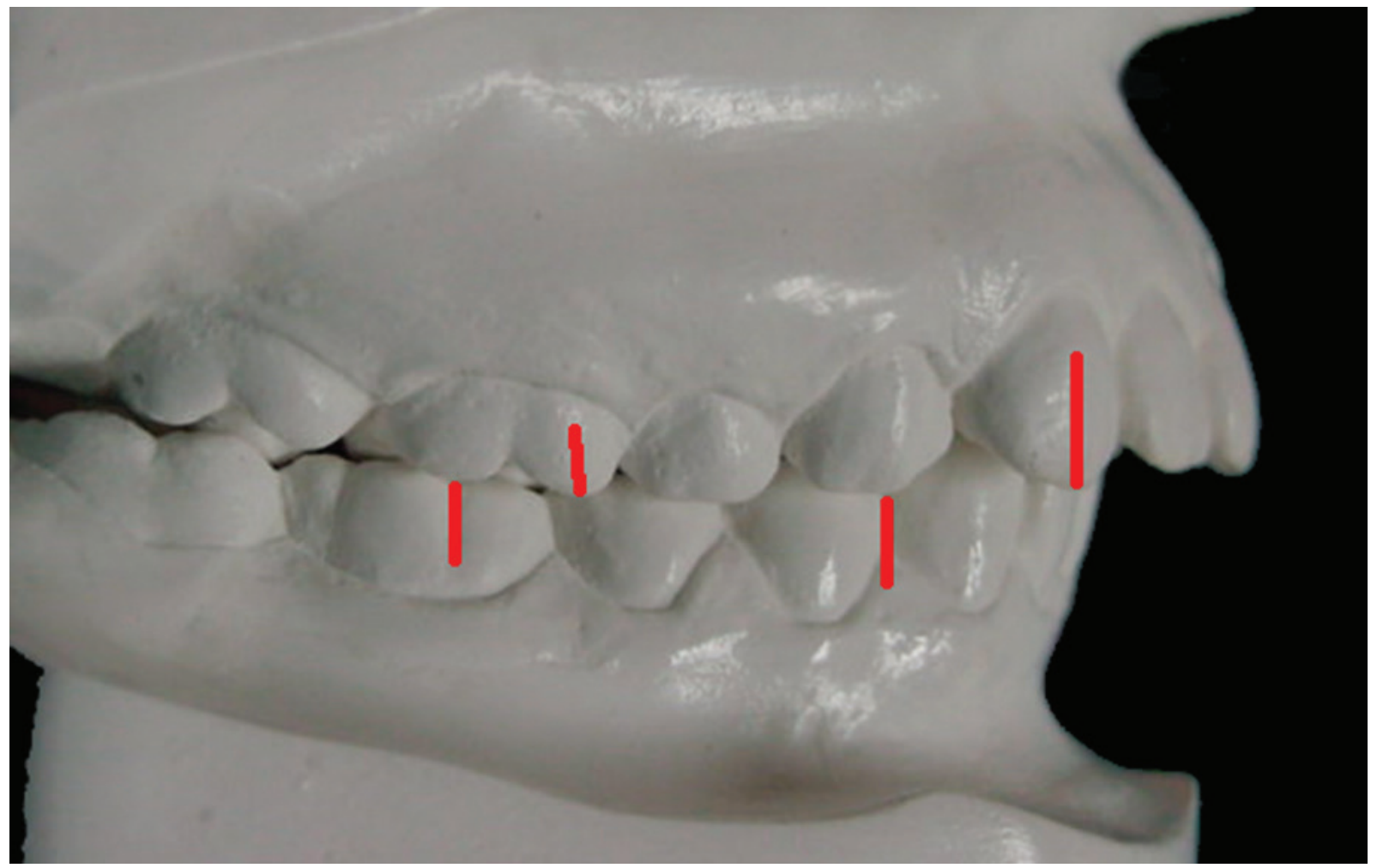

Figura 7: Apresenta a relação molar de Classe II e de caninos em c2.

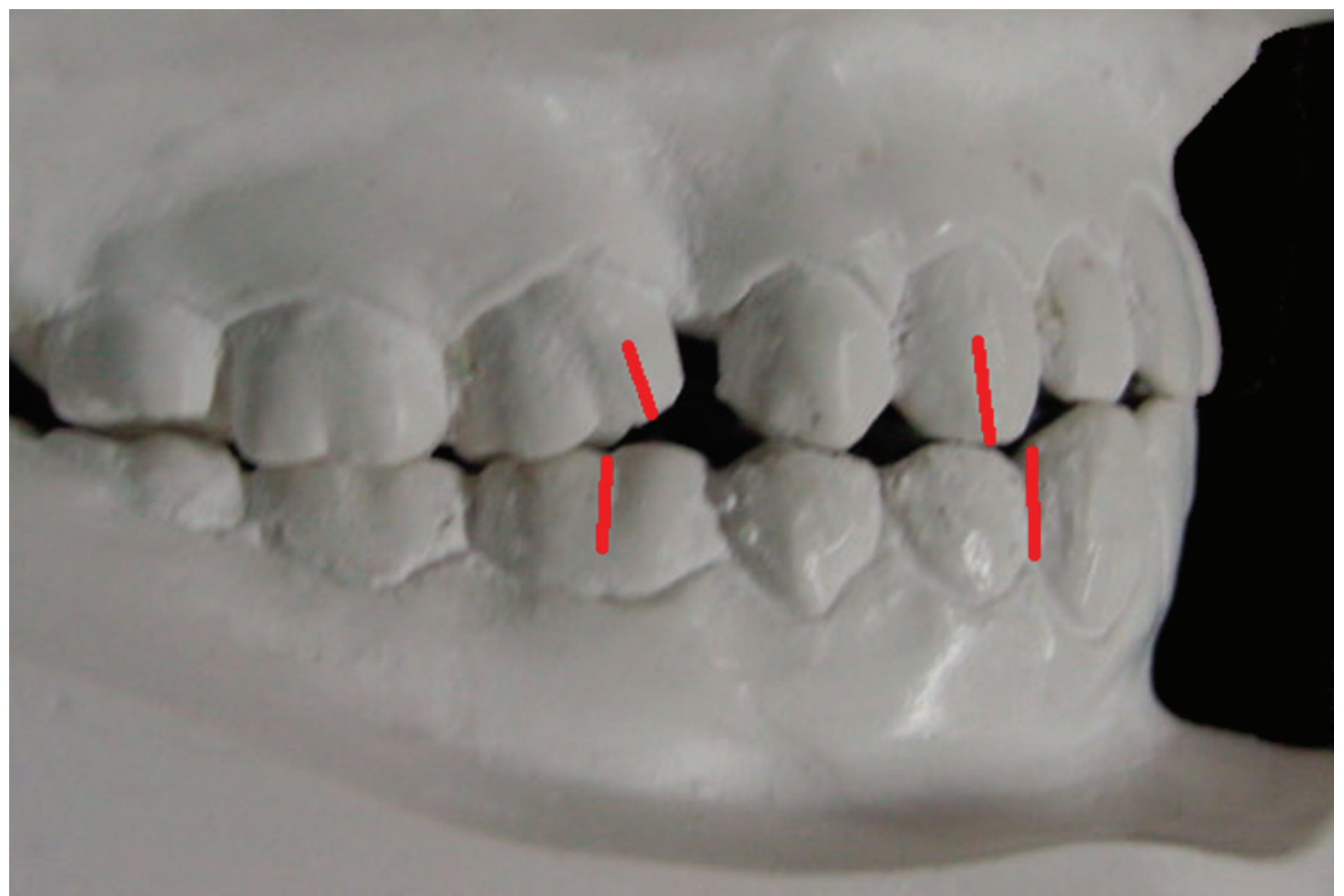

Figura 8: Apresenta a relação molar de Classe II e de caninos em c3. 


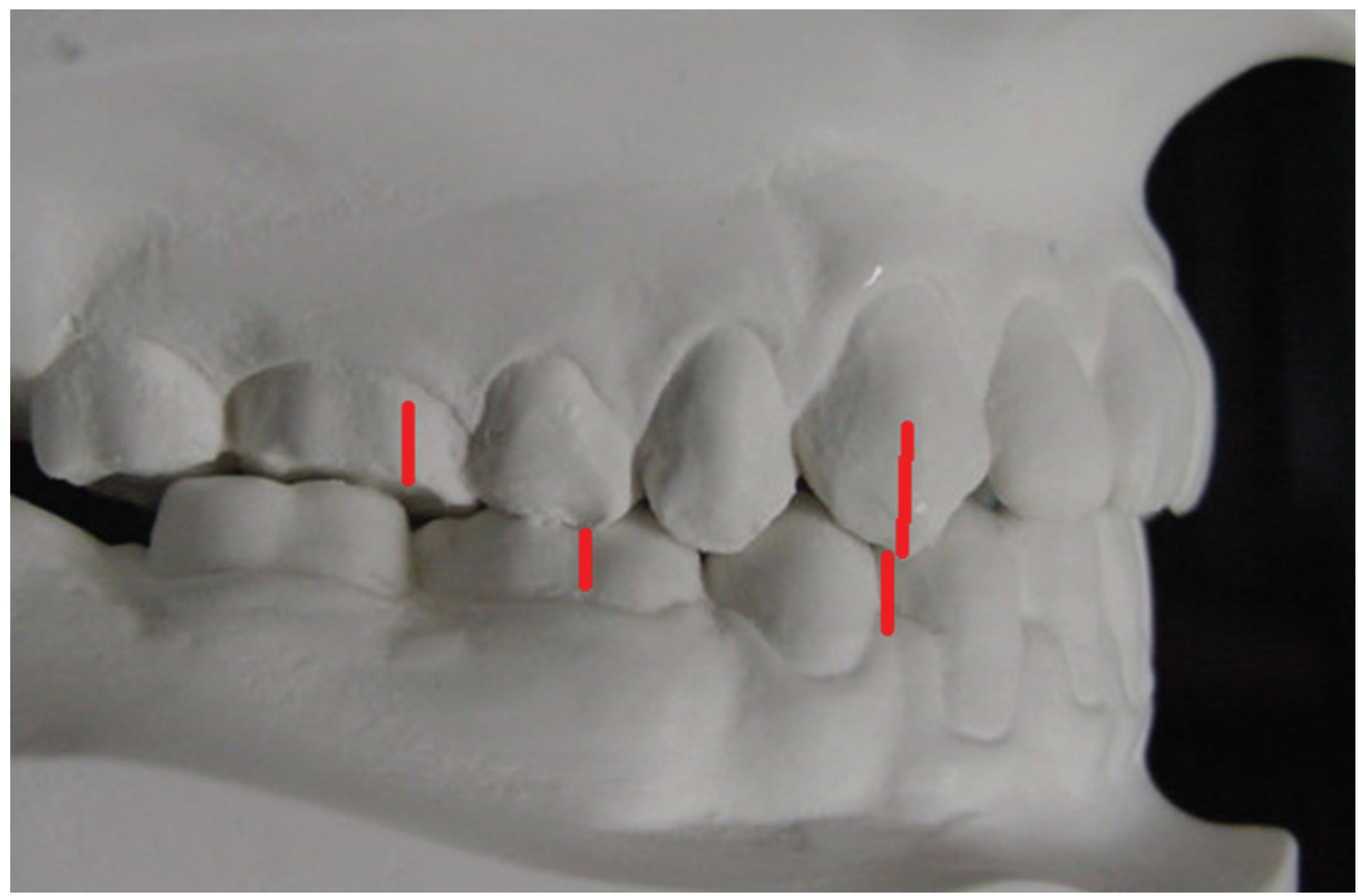

Figura 9: Mostra a relação molar de Classe III e de caninos em c1.

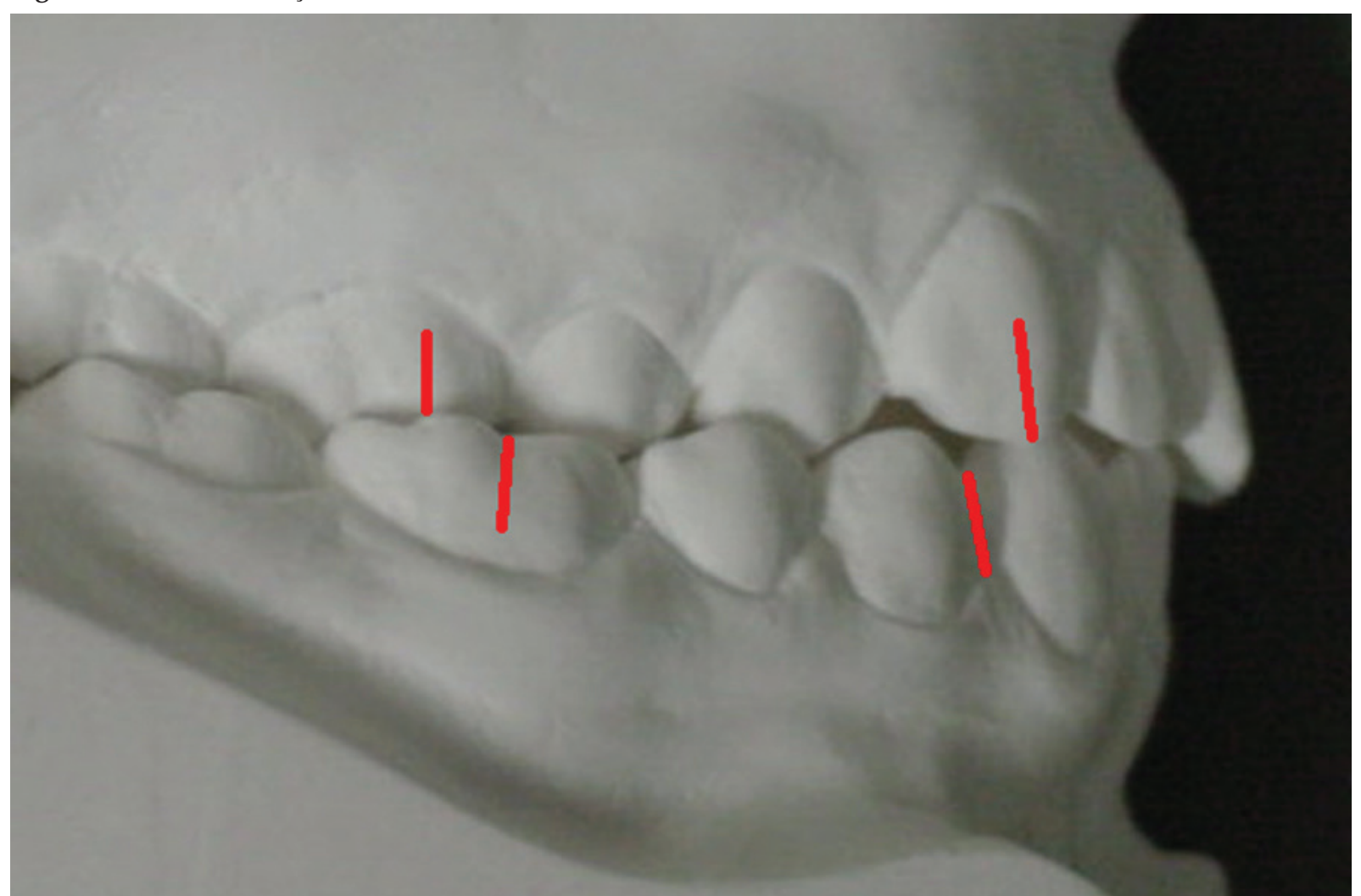

Figura 10: Mostra a relação molar de Classe III e de caninos em c2. 


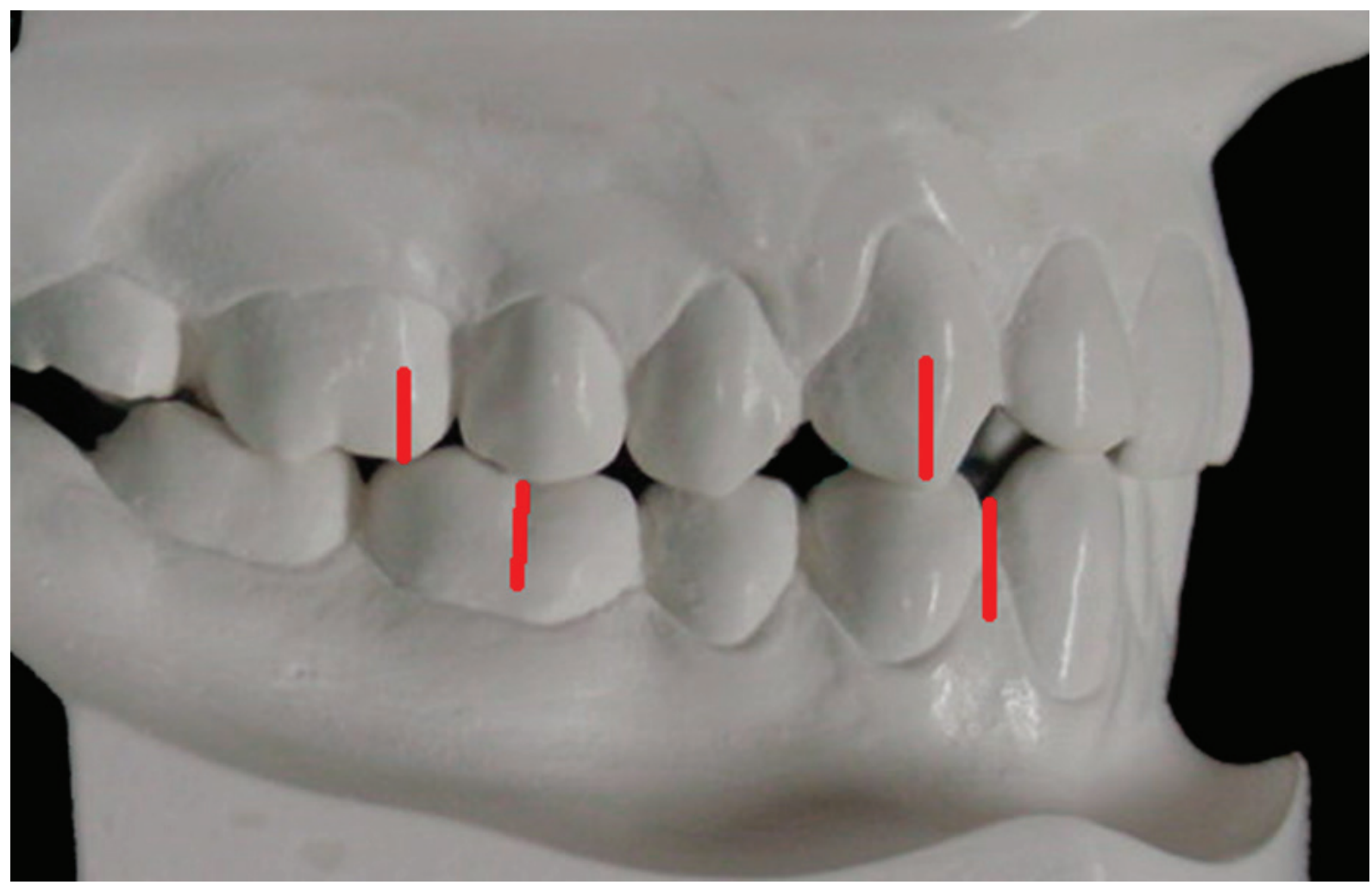

Figura 11: Mostra a relação molar de Classe III e de caninos em c3.

\section{DISCUSSÃO}

Angle (1899) introduziu a classificação das más oclusões considerando a relação mesiodistal dos elementos dentários. Sua nomenclatura fundamentava-se no princípio de que os primeiros molares superiores ocupavam uma posição imutável e, portanto, foram estabelecidos como a chave para a determinação das terminologias em Classe I, Classe II e Classe III, empregando-se algarismos romanos e 1 e 2 para diferenciar os tipos de Classe II, porém, com algarismos arábicos ${ }^{4}$.

Apesar da classificação de Angle ser aceita mundialmente, a pressuposição de que os primeiros molares superiores eram imutáveis, impedia que dentes em mesioversão, dentes impactados, dentes ausentes, más posições dentárias individuais, más posições envolvendo os planos transversais e verticais, e dentes decíduos fossem classificados, constituindo-se em motivos de críticas $^{13}$. A posição natural dos primeiros molares permanentes se altera na dentadura mista, com o desenvolvimento da oclusão, com o crescimento craniofacial e também sofre influência do espaço livre de Nance ${ }^{14}$.

Outro aspecto referente a classificação de Angle é a inconsistência quando profissionais analisam as más oclusões em fotografias ou modelos de gesso ${ }^{1}$.Assim sendo, outras classificações foram propostas na Ortodontia e que contemplasse a inclusão de outros elementos dentários, além dos primeiros molares ${ }^{3,5-10}$. A posição correta dos incisivos e sua influência na estética do sorriso e da face desperta mais interesse dos profissionais do que a posição dos primeiros molares ${ }^{1}$. O canino superior é considerado um dos dentes mais estáveis pelo seu comprimento total e por se encontrar bem ancorado no osso basal, 
proporcionando a proteção durante os movimentos mandibulares excursivos de lateralidade. Assim sendo, considerando-se a importância deste elemento dentário na oclusão e pela posição estratégica que ocupa no arco dentário, é que se justifica a proposição de uma classificação baseada em sua posição sagital, uma vez que a simples utilização de uma nomenclatura baseada em primeiros molares possibilitou classificar apenas $7 \%$ de uma amostra de 4309 modelos de gesso do estudo de Broadbent-Bolton ${ }^{15}$.

Os profissionais devem analisar criteriosamente as características de uma má oclusão, e somente após um estudo minucioso do problema, é que se deve classificá-la. Uma vez que a classificação deve ser empregada analisando-se todos os elementos dentários do que simplesmente observar como os molares se intercuspidam, pois a precisão na análise é fundamental para o diagnóstico e para planejar a oclusão final ${ }^{111}$. Ressalta-se que a relação de incisivos e caninos é fator determinante para se definir uma oclusão ideal ${ }^{16}$. Portanto, a classificação proposta amplia a possibilidade diagnóstica, contribuindo para o estabelecimento de um plano de tratamento adequado, visando o posicionamento final dos caninos ${ }^{1}$.

\section{CONCLUSÃO}

A rotulação imediata da má oclusão pode induzir ao erro, principalmente quando se classifica simplesmente pela relação dos primeiros molares. A classificação pela posição sagital de caninos complementando a classificação de Angle amplia a possibilidade de diagnóstico e de elaboração de um plano de tratamento mais adequado, considerando-se a importância deste elemento dentário para a oclusão.

\section{REFERÊNCIAS BIBLIOGRÁFICAS.}

1. Katz MI. Angle classification revisited 1: is current use reliable? Am J Orthod Dentofac Orthop 1992; 102(2): 173-9.

2. Organização Mundial de Saúde. Oral health surveys: basic methods - 5 th ed. (C World Health Organization 2013; Levantamentos em saúde bucal: métodos básicos - 5 a ed. (C) Faculdade de Odontologia Universidade de São Paulo (FOUSP) 2017. [cited MaY 13]. Available from: URL: http://www.fo.usp.br/wp-content/uploads/2011/06/Levantamentos-Saude-Bucal_5ed_Nov2017.pdf.

3. Horowitz SL, Hixon EH. The nature orthodontic diagnosis. St Louis: CV Mosby, 1996: 325-43.

4. Angle EH. Classification of malocclusion. Dent Cosmos 1899; v.41:248-264, 350-357.

5. Case CS. The teaching of orthopedic dentistry. Dent Items Interest 1904; 26:481-

6. Lischer BE. Principles and methods of Orthodontics. Philadelphia: Lea and Febiger; 1912.

7. Dewey M. Classification of malocclusion. Int J Orthod 1915; 1(3): 133-47.

8. Anderson GM. Practical orthodontics. $9^{\text {th }}$ ed. St Louis: CV Mosby 1960; 114-50.

9. Ackerman JL, Proffit WR. The characteristics of malocclusion: a modern approach to classification and diagnosis. Am J Orthod Dentofac Orthop 1969; 56(5): 443-54.

10. Katz MI. Angle classification revisited 2: a modified Angle classification. Am J Orthod Dentofac Orthop 1992; 102(3): 277-84.

11. Moyers RE. Ortodontia, 4aㅡ ed. Rio de Janeiro: Guanabara Koogan; 1991.

12. Foggiato AA. Classificação das maloclusões dos caninos. Dissertação (Mestrado em Ortodontia) - Centro de Pesquisas Odontológicas São Leopoldo Mandic, Campinas, 2002.

13. Mageet AO. Classification of skeletal and dental malocclusion: revisited. Stoma Edu J 2016; 3(2): 38-44.

14. Arya BS, Savara BS, Thomas DR. Prediction of first molar occlusion. Am J Orthod Dentofac Orthop 1973; 63(6): 610-21.

15. Hans MG, Broadbent BH, Nelson SS. The Broadbent -Bolton study past, present and future. Am J Orthod Dentofac Othop 1994; 105(6): 598-603.

16. Andrews, L F. The six keys to normal occlusion. Amer. J. Orthod. n.62, p.296-309, 1972. 